\title{
Dilated cardiomyopathy in children: determinants of outcome
}

Michael Burch, Shahab A Siddiqi, David S Celermajer, Charmaine Scott, Catherine Bull, John E Deanfield

\begin{abstract}
Objective-To determine the outcome of dilated cardiomyopathy presenting in childhood and the features that might be useful for prognostic stratification.

Setting-Supraregional paediatric cardiology unit.

Design-Retrospective analysis.

Background-The natural history of dilated cardiomyopathy in children is not well characterised. Previous studies have shown a variable relation between age at presentation and outcome, and sudden death has been infrequent.

Methods-Retrospective study of 63 consecutive patients with idiopathic dilated cardiomyopathy presenting between 1979 and 1992. Survival curves were constructed by the Kaplan-Meier method.

Results-Age at diagnosis ranged from 1 day to 15 years (median 12 months) and follow up ranged from 1 day to 13 years (median 19 months). Actuarial survival from presentation was $79 \%$ at one year (95\% confidence interval (95\% CI) $66 \%-88 \%)$ and $61 \%(44 \%-74 \%)$ at five years. Univariate analysis showed that mural thrombus, left ventricular end diastolic pressure $>20 \mathrm{~mm} \mathrm{Hg}$, and age at presentation $>2$ years were predictors of adverse outcome, but on multivariate analysis only age at presentation was significant. Left ventricular echocardiographic indices either did not improve or deteriorated in 36 children (17 of whom died, four suddenly, and three were transplanted), partially improved in 16 (three of whom died, all suddenly), and returned to normal in 11 (all of whom have survived).

Conclusions-Older age at presentation and lack of improvement in systolic function are associated with an adverse outcome, and early transplantation should be considered in these patients. There is a persistent risk of late sudden death in those children in whom echocardiographic dimensions remain abnormal.
\end{abstract}

(Br Heart F 1994;72:246-250)

Idiopathic dilated cardiomyopathy in adults has a poor prognosis, with about $40 \%$ of patients dying within two years of diagnosis. ${ }^{1-3}$ The main risk factor for early death is a profoundly decreased ejection fraction. ${ }^{12}$ In adults, sudden death is also common and may occur late after presentation even in patients whose heart failure is well controlled. ${ }^{2-5}$ The natural history of dilated cardiomyopathy in children is less well characterised and, in particular, the effect of age at presentation on prognosis is controversial. Griffin et al suggested a better outcome for patients $<2$ years of age, ${ }^{6}$ but this has not been found by others. ${ }^{7-9}$ The advent of successful paediatric heart transplantation has given clinicians a new therapeutic option: our actuarial survival at one and three years after transplantation for dilated cardiomyopathy is currently $85 \%$ in our hospital (M de Leval, P Rees, personal communication). Patient selection and timing of surgery, however, has not been well defined. Survivors considered too well for transplantation may, like their adult counterparts, remain at risk of late sudden death, but this is thought to be a rare event in childhood. ${ }^{10}$ We have re-examined the natural history of idiopathic dilated cardiomyopathy in children to define high risk subgroups who may benefit from transplantation or more active measures to prevent sudden death.

\section{Patients and methods}

PATIENTS

The records of all patients presenting with dilated cardiomyopathy to the Hospital for Sick Children, Great Ormond Street from January 1979 to January 1992 were reviewed. The entry point of 1979 was chosen as this ensured that all patients had an echocardiographic assessment of left ventricular dimensions and function and enabled consistent entry criteria to be applied-that is, a shortening fraction of $\leqslant 25 \%$ and a left ventricular internal diameter of $>95$ th centile for age. ${ }^{11}$ The measurements used were obtained at the time of assessment. Excluded as secondary cardiomyopathies were storage disease, birth asphyxia, anthracycline toxicity, connective tissue disease, incessant arrhythmias, mitochondrial myopathy, and familial calcific arterial stenosis. No evidence of neuromyopathic disease developed during the study period in any of the patients with dilated cardiomyopathy. Familial dilated cardiomyopathy was seen in seven patients $(11 \%)$. This was obtained from the family history as prospective echocardiographic screening was not performed. These patients were not referred because of suspected familial cardiomyopathy. Recently we have also excluded Lyme disease myocarditis. An anomalous coronary artery was excluded echocardiographically. 
Viral myocarditis was excluded by paired serology. A history of recent viral illness in childhood was thought to be too common to be considered evidence of myocarditis. The first heart was transplanted at our hospital in 1987 and no patient in this series was referred primarily for transplantation. Decisions about treatment were at the discretion of the patient's physician. Since the mid 1980s, angiotensin converting enzyme inhibitor treatment (captopril) has been widely used starting soon after admission to hospital. Since 1988 anticoagulation has been used routinely for patients with persistent left ventricular dysfunction.

\section{MEASUREMENTS}

We defined improvement in left ventricular echocardiographic indices as either an increase in fractional shortening of $\geqslant 10 \%$ or a return of left ventricular end diastolic dimensions to a value $<95$ th centile for age. When such an improvement occurred patients were generally considered too well for transplantation. If the fractional shortening became $>25 \%$, and the end diastolic dimension $<95$ th centile, we defined this as a return to normal. The date when these indices were first recorded was taken as the date of improvement or return to normal. Cardiac catheterisation was performed in 17 patients, and was usually undertaken as part of a transplantation assessment. Continuous ambulatory electrocardiograms were monitored at the discretion of the attending physician in 21 of $63(33 \%)$ patients.

\section{STATISTICS}

Survival curves were constructed by the Kaplan-Meier method. ${ }^{12}$ Fischer's exact test was used for univariate analysis. Cox's proportional hazards method was used for risk factor analysis, ${ }^{13}$ and relative risks and survival probabilities are quoted with $95 \%$ confidence intervals $(95 \% \mathrm{cls})$. To be consistent with earlier reports, ${ }^{9}$ patients undergoing transplantation were withdrawn from our study alive at the time of transplant. Late sudden death was defined as unexpected mortality $>3$ months from presentation in a child with well controlled heart failure.

The echocardiographic definitions we have outlined allowed us to examine the changing proportion over time from presentation of patients who were in three mutually exclusive categories: dead with abnormal fractional shortening, alive with continuing abnormal fractional shortening, and alive with fractional shortening that has returned to normal (none of those whose fractional shortening returned to normal died). Death without return to normal and normal are treated as absorbing states (death is a permanent condition of being unable to become normal. Patients who die were censored beyond the time frame of interest to the study). These categories were stratified by age of presentation below and above two years.

For the subset of patients whose fractional shortening improved beyond the level at which transplantation would be envisaged, we also examined the changing proportion over time in three mutually exclusive categories: deaths (all sudden) in patients who had continuing impairment, alive with continuing impairment, and normal. Once again, the effects of deaths on the normal curve and vice versa was examined by treating both as "absorbing states". As echocardiographic improvement was time dependent, it could not be incorporated into the multivariate analysis.

\section{RESULTS}

Median age at diagnosis of the 63 children was 12 months (range 1 day to 15 years); 37 of $61(61 \%)$ were aged $<2$ years and $24(39 \%)$ were $>2$ years. Median follow up was 19 months, range 1 day (death shortly after presentation) to 13 years.

\section{HEMODYNAMICS}

Cardiac catheterisation was performed in 17 children. These were in symptomatic heart failure and had generally not shown an improvement in echocardiographic dimensions. Catheterisation was undertaken as part of transplantation assessment at two weeks to six years (median two months) from presentation. Left ventricular end diastolic pressure was $>20 \mathrm{~mm} \mathrm{Hg}$ in six patients: of these four died and two were transplanted. Death or transplantation was less common $(P<0.05)$ in the 11 with pressures $<20 \mathrm{~mm} \mathrm{Hg}$; there were no transplants and only two deaths (one sudden and the other from low cardiac output).

\section{AMBULATORY ELECTROCARDIOGRAPHIC}

MONITORING

Ambulatory monitoring was performed in 21 of $63(33 \%)$ between two months and eight years (median eight months) after presentation and repeated in 13 of $21(62 \%)$. Nonsustained ventricular tachycardia $(<30 \mathrm{~s})$ was documented in four patients, one of whom had previously had symptomatic atrial flutter. All were treated: amiodarone was used in three and disopyramide in one. Three of these patients died suddenly. In one, amiodarone had been withdrawn one year before death after normal ambulatory monitoring. Sudden death was significantly more common in the group with ventricular arrhythmia (3/4) than with normal rhythm $(1 / 18, \mathrm{P}<0.05)$.

\section{ECHOCARDIOGRAPHIC PATTERN OF} IMPROVEMENT AND RELATION TO LATE SUDDEN DEATH

Fractional shortening at presentation ranged from $5 \%-20 \%$ (median $11 \cdot 5 \%$ ). The pattern of improvement is shown in the multistate survival curves (figs 1 and 2) and in the flow diagram (fig 3). Three groups were defined:

(a) No documented improvement: 36 patients had no documented improvement in echocardiographic indices. This group includes the seven patients who died $<3$ months from presentation and the 29 that survived $>3$ months, 10 of whom subsequently 
Figure 1 Summary of outcomes: patients are presented in three mutually exclusive categories (A) patients $<2$ yr at presentation. (B) patients $\geqslant 2$ yr at presentation.

Figure 2 Summary of outcome for the subset of patients whose ventricle improved beyond the level where transplantation would be envisaged: patients are presented in three mutually exclusive categories.
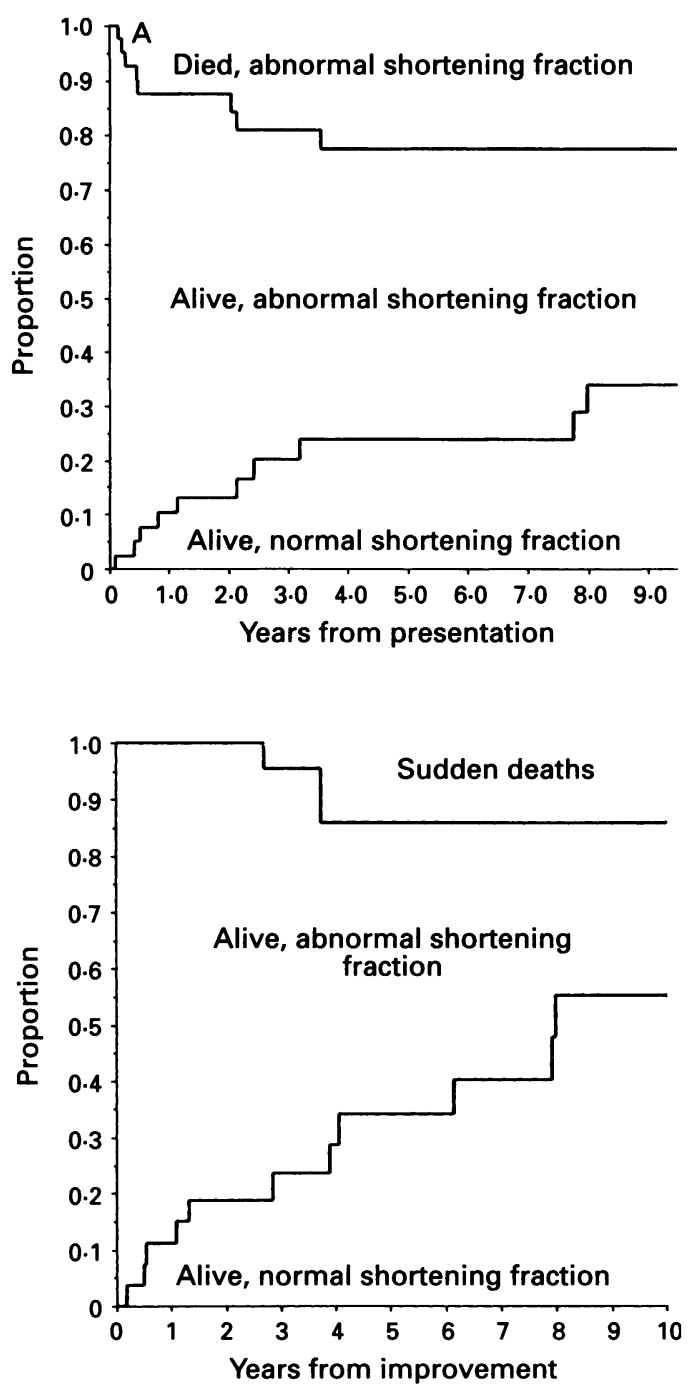

died (four suddenly) and three were transplanted.

(b) Documented improvement: 16 children had documented improvement in fractional shortening (and were considered too well for transplantation). No such patient has deteriorated again or died from heart failure, but three have died suddenly (fig 2 ).

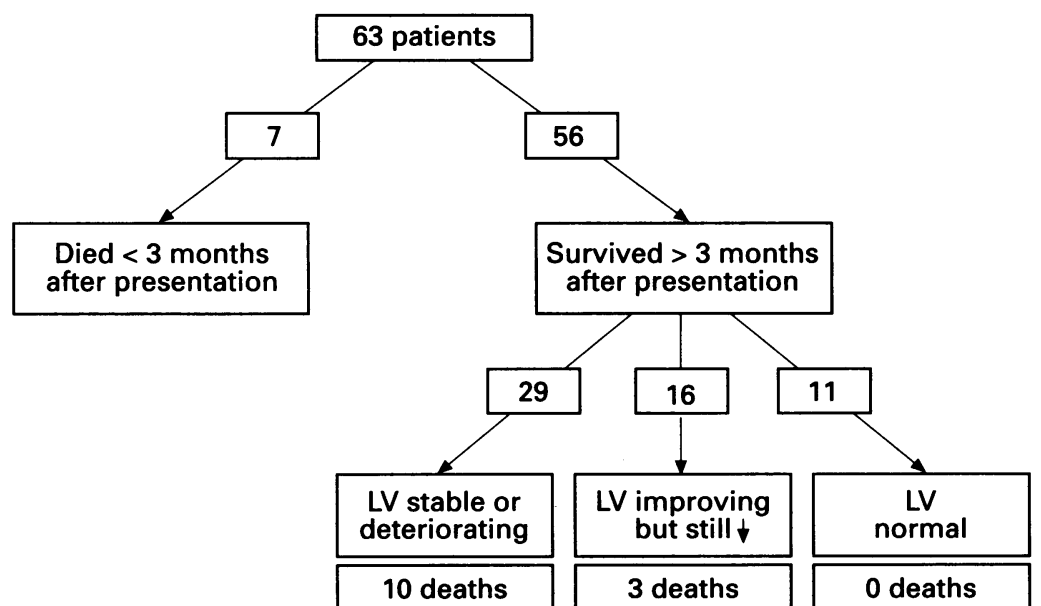

Figure 3 Flow diagram representing outcome and pattern of improvement in 63 patients with dilated cardiomyopathy.

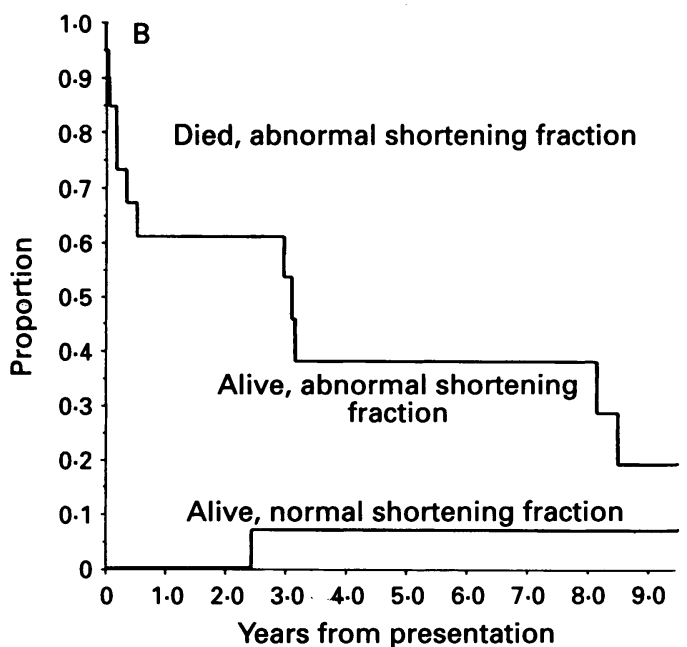

(c) Return to normal dimensions: In 11 children ventricular function continued to improve to within normal limits and none has died to date. Most (10/11: $91 \%)$ of this subgroup were $<2$ years old at presentation (fig 1).

SURVIVAL ANALYSIS

Actuarial survival from presentation was $79 \%$ $(95 \% \mathrm{CI}, 66 \%-88 \%)$ at one year and $61 \%$ $(44 \%-74 \%)$ at five years (fig 4$)$.

On univariate analysis, both age and presentation $>2$ years and mural thrombus were predictors of adverse outcome at one year ( $P$ $<0.05)$. The presence of mural thrombus was not associated with fractional shortening, nor with regional dyskinesis. Of the factors explored as predictors of survival (table) on multivariate analysis, only age at presentation of $<2$ years was significant (fig 1). Patients who presented aged $>2$ years had a relative risk of death of 4.67 (95\% CI, 1.72-12.72) compared with those who presented at $<2$ years of age.

\section{POSTMORTEM DATA}

A postmortem examination was performed in 11 cases. The coronary arteries were normal in all. In two infants there was mild endocardial fibroelastosis, but this was not associated with ventricular hypertrophy and was not thought to be primary endocardial fibroelastosis. Histological examination did not show evidence of inflammatory cells and storage diseases were excluded.

\section{Discussion}

Despite the highly variable course of idiopathic dilated cardiomyopathy in children, this study has identified a number of easily obtained clinical indices that can be used to rationalise management of these patients. Although exploring the presenting features that relate to mortality is important, understanding the pattern of improvement in ventricular function is equally crucial to decision making, particularly for transplantation referral. 
Figure 4 Survival curve from presentation of 63 children with dilated cardiomyopathy. The number followed up are indicated at intervals above the horizontal axis. The interrupted lines represent $95 \%$ CIs.

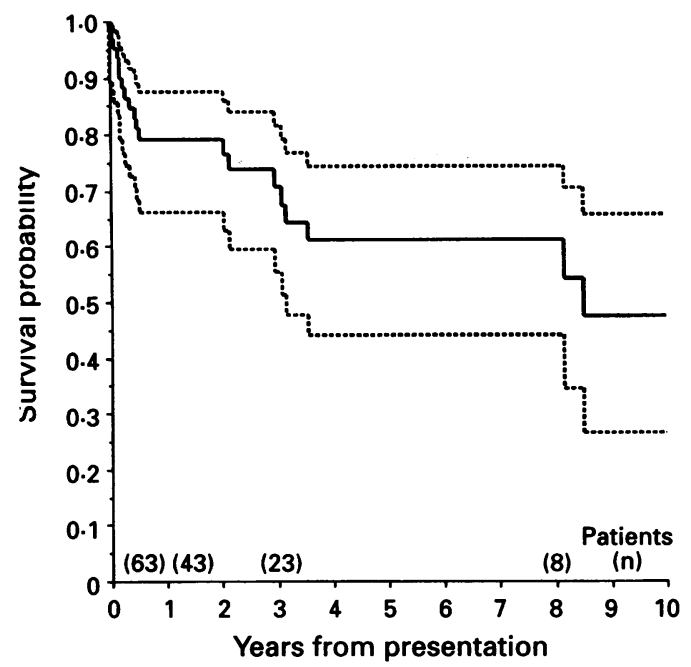

Deaths from cardiac failure in patients with dilated cardiomyopathy could theoretically be reduced by transplantation. Our data have shown that the outcome of children in the subgroup with no documented improvement in left ventricular echocardiographic dimensions is poor, with death principally from cardiac failure, and such patients should be considered for transplantation. This decision must be made early as over half of the deaths from cardiac failure occurred within three months of presentation. Other factors that would influence early referral were mural thrombus, left ventricular end diastolic pressure $>20 \mathrm{~mm} \mathrm{Hg}$, and age at presentation of $>2$ years.

A second subgroup of patients identified by this study consisted of children in whom the left ventricular echocardiographic indices had improved to the extent that they were considered too well for transplantation. None of these patients subsequently deteriorated with worsening cardiac failure; nevertheless late deaths did occur and all were sudden. Previous research has not identified late sudden death as a problem in dilated cardiomyopathy in childhood..$^{10}$ In our series sudden death accounted for more than half of all deaths $>3$ months after presentation, but only occurred in patients in whom left ventricular function remained abnormal. It may be possible to reduce significantly the high incidence of sudden death by combining more detailed arrhythmia surveillance with invasive electrophysiological studies and aggressive treatment of any documented or inducible arrhythmias, a strategy that has improved survival in hypertrophic cardiomyopathy ${ }^{14}$ and in paediatric survivors of near miss sudden death, ${ }^{15}$ but not in children with tetralogy of Fallot. ${ }^{16}$ Sudden death occurred despite treatment with amiodarone in three patients and a proarrhythmic effect cannot be excluded in these children. No evidence of heart block was seen on ambulatory monitoring or resting electrocardiograms, and there was no evidence to suggest bradycardia as a mode of sudden death.

A third group of children were those in
Multivariate analysis with the Cox proportional hazards model for survival from presentation (63 patients (20 deaths))

\begin{tabular}{|c|c|c|}
\hline & Patients ( $n$ ) & $\begin{array}{l}\text { relative (95\% CIs) } \\
\text { risk }\end{array}$ \\
\hline $\begin{array}{l}\text { Age at presentation: } \\
<2 \mathrm{yr} \\
\geqslant 2 \mathrm{yr}\end{array}$ & $\begin{array}{l}38 \\
25\end{array}$ & ${ }_{4.67}^{1}\left(1.72\right.$ to $\left.12.72^{\star}\right)$ \\
\hline $\begin{array}{l}\text { Mural thrombus: } \\
\text { Absent } \\
\text { Present }\end{array}$ & $\begin{array}{r}54 \\
9\end{array}$ & 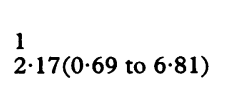 \\
\hline $\begin{array}{l}\text { Captopril: } \\
\text { Not given } \\
\text { Given }\end{array}$ & $\begin{array}{l}32 \\
31\end{array}$ & $1.19(0.43$ to 3.33$)$ \\
\hline $\begin{array}{l}\text { Parenteral inotropes: } \\
\text { Not given } \\
\text { Given }\end{array}$ & $\begin{array}{l}49 \\
14\end{array}$ & $1.76(0.53$ to 5.8$)$ \\
\hline $\begin{array}{l}\text { Family history: } \\
\text { None } \\
\text { Present }\end{array}$ & $\begin{array}{r}56 \\
7\end{array}$ & $\begin{array}{l}1 \\
0.42(0 \cdot 10 \text { to } 1 \cdot 78)\end{array}$ \\
\hline $\begin{array}{l}\text { FS at presentation: } \\
10 \%-25 \% \\
<10 \%\end{array}$ & $\begin{array}{l}33 \\
30\end{array}$ & $1.82(0.5$ to 6.3$)$ \\
\hline
\end{tabular}

whom echocardiographic dimensions returned to normal. Such patients were not at risk of sudden death, and the families of these children can be cautiously reassured.

Multivariate analysis showed young age at presentation to be a predictor of survival. Similar findings have been reported previously by Griffin et $a l^{6}{ }^{6}$ who also studied a young population (median age $<2$ years). Studies that did not find this age related difference in outcome investigated mainly older children. The better outlook for infants is fortuitous as the donor pool for infant transplantation is limited, ${ }^{17}$ and the results of transplantation in infancy are worse than in older children and adults. ${ }^{18}$

There may be several explanations for the better outcome in younger children. The aetiological agent may differ; immunologically mediated myocardial injury may be less common. ${ }^{19}$ Alternatively, infants may have a cardiovascular system that copes better with severe myocardial damage, as infant myocytes retain the propensity to regenerate, ${ }^{20}$ or afterload may be lower due to more compliant peripheral arteries.

Idiopathic dilated cardiomyopathy is a broad term that is likely to encompass a heterogeneous aetiology, thus care must be taken in extrapolating these results. This study is from a tertiary referral centre and the population may be biased towards the more severely affected children, but none were referred exclusively for transplantation. Also, it is unusual for a child with suspected dilated cardiomyopathy to be managed entirely at a district hospital, without involvement of a paediatric cardiologist. Patients who had positive viral serology were excluded but biopsies were not performed. This seems justified because the diagnosis of viral myocarditis is difficult even when biopsies are taken ${ }^{21}$ and Coxsackie myocarditis and dilated cardiomy- 
opathy may be a continuum. ${ }^{22}$ Furthermore, these patients need similar decisions to idiopathic cardiomyopathy regarding transplantation. As only three patients had evidence of a recent viral infection during the study period, their inclusion in the survival analysis would not have greatly altered the results.

In common with all retrospective studies, the data are subject to the constraints of preexisting departmental policies. For example, in this study the presence of ventricular arrhythmias on ambulatory monitoring was associated with late risk of sudden death, but the data were incomplete. The value of ambulatory monitoring in predicting sudden death is uncertain in adults with dilated cardiomyopathy, ${ }^{2324}$ and in children with congenital heart disease. ${ }^{16}$ Also, a more precise assessment of ventricular function would have been desirable as fractional shortening will not reflect segmental wall motion abnormalities and may be altered by changes in afterload. Despite the limitations of the method, it seems that by the use of large $(>10 \%)$ changes in fractional shortening, in conjunction with left ventricular end diastolic dimension, helpful deductions can be made on the course of dilated cardiomyopathy in children. In future studies it would be of interest to correlate our assessment of fractional shortening (and echocardiographic ejection fraction) with the isotopic methods of assessment of ejection fraction that are more commonly used in adult practice. ${ }^{12}$

In conclusion these data show that the prognosis is poor in children with dilated cardiomyopathy, but the mortality may be reduced if early transplantation is considered for those presenting at $>2$ years if no improvement in echocardiographic indices is shown, particularly if mural thrombus is present or left ventricular end diastolic pressure is $>20$ $\mathrm{mm} \mathrm{Hg}$. Survivors remain at risk of sudden death until echocardiographic dimensions return to normal.

1 Keogh AM, Freund J, Baron DW, Hickie JB. Timing of cardiac transplantation in idiopathic dilated cardiomyopathy. Am F Cardiol 1988;61:418-22.

2 Hofman T, Meinertz T, Kasper W, et al. Mode of death in idiopathic dilated cardiomyopathy: a multivariate analysis of prognostic determinants. Am Heart $\mathcal{f}$ 1988;116: 1455-63.

3 Caforio ALP, Stewart JT, McKenna WJ. Idiopathic dilated cardiomyopathy. $B M F$ 1990;300:890-1.

4 Van Olhausen K, Stienen U, Schwarz F, Kabler W, Maye J. Long term prognostic significance of ventricula arrhythmias in idiopathic dilated cardiomyopathy. $A m \mathcal{F}$ Cardol 1988;61:146-51.

5 Packer M. Sudden unexpected death in patients with congestive heart failure: a second frontier. Circulation 1985;72:681-5.

6 Griffin ML, Hernandez A, Martin TC, et al. Dilated cardiomyopathy in infants and children. $\mathcal{F} \mathrm{Am}$ Coll Cardiol 1988;11:139-44.

7 Akagi T, Benson LN, Lightfoot NE, Clin K, Wilson G, Freedom RM. Natural history of dilated cardiomyopaFreedom RM. Natural history of dilated cardion

8 Chen SC, Nouri S, Balfour I, Jureidini S, Appleton RS Clinical profile of congestive cardiomyopathy in children. F Am Coll Cardiol 1990;15:189-93.

9 Lewis AB, Chabot M. Outcome of infants and children with dilated cardiomyopathy. $\mathrm{Am} f \mathrm{f}$ Cardiol $1991 ; 68: 365-9$.

10 Friedman RA, Moak JP, Garson A. Clinical course of idiopathic dilated cardiomyopathy in children. $7 \mathrm{Am}$ Coll Cardiol 1991;18:152-6.

11 Henry WL, Ware J, Gardin JM, Hepner SI, McKay J, Weiner M. Echocardiographic measurements in normal subjects: growth-related changes that occur between infancy and early adulthood. Circulation 1978;57:278.

12 Kaplan EL, Meier P. Nonparametric estimation from incomplete observations. Fournal of the American incomplete observations. Fournal

13 Cox M. Analysis of survival data. London: Chapman and Hall, 1984.

14 Fananapazir L, Chang AC, Epstein SE, McCreavey D. Prognostic determinants in hypertrophic cardiomyopathy: prospective evaluation of a therapeutic strategy based on clinical, Holter, hemodynamic, and electrophysiological findings. Circulation 1992;86:730-40.

15 Silka M, Kron J, Walance CG, Culter JE, McAnulty JH Assessment and follow up of pediatric survivors of sud den cardiac death. Circulation 1990;82:341-9.

16 Deanfield JE. Late ventricular arrhythmias occurring after repair of tetralogy of Fallot: do they matter? Int $\mathcal{F}$ Cardiol 1991;30:143-50.

17 Stuart AG, Wren C, Sharples PM, Hunter S, Hey EN. Hypoplastic left heart syndrome: more potential transHypoplastic left heart syndrome: more potential trans-
plant recipients than suitable donors. Lancet plant recipients

18 Kaye MP. The Registry of the International Society for heart lung transplantation: 9th Official Report-1992. F Heart Lung Transplant 1992;11:599-606.

19 Caforio ALP, Bonifacio E, Stewart JT, et al. Novel organspecific circulating cardiac autoantibodies in dilated ca diomyopathy. 7 Am Coll Cardiol 1990;15:1527-34.

20 Olivetti G, Anversa P, Load A. Morphometric study of early postnatal developments in the left and right ventricular myocardium of the rat Circ Res 1980;46:503-12.

21 Hingorani $\mathrm{AD}$, Dollery CT, Oakley CM, Bloom SR, Cohen J. Post infectious myocarditis: still a largely clinical diagnosis. $B M 7$ 1992;304:1676-8.

22 Jin $\mathrm{O}$, Sole MJ, Butany JW, Chia WK, McLaughlin PR, Liu P, et al. Detection of entero virus RNA in myocardial biopsies from patients with myocarditis and cardiomyopathy using gene amplification by polymerase diomyopathy using gene amplification

23 Huang SK, Messer JV, Denes P. Significance of ventricular tachycardia in idiopathic dilated cardiomyopathy: observations in 35 patients. $A m \mathcal{F}$ Cardiol 1983;51:507-12.

24 Olhausen K, Stienen U, Schwarz F, Kubler W, Meyer Long term prognostic significance of ventricular arrhythmias in idiopathic dilated cardiomyopathy. Am $\mathcal{f}$ Cardiol 1988;61:146-51. 